\title{
A presentation model for multimedia summaries of behavior
}

\author{
Martin Molina, Victor Flores \\ Department of Artificial Intelligence, \\ Universidad Politécnica de Madrid, \\ Campus de Montegancedo S/N 28660 \\ Boadilla del Monte, Madrid, Spain. \\ martin.molina@upm.es, vflores@fi.upm.es
}

\begin{abstract}
Presentation models are used by intelligent user interfaces to automatically construct adapted presentations according to particular communication goals. This paper describes the characteristics of a presentation model that was designed to automatically produce multimedia presentations about the summarized behavior of dynamic systems. The presentation model is part of the MSB application (Multimedia Summarizer of Behavior). MSB was developed for the problem of management of dynamic systems where different types of users (operators, decision-makers, other institutions, etc.) need to be informed about the evolution of the system, especially during critical situations. The paper describes the details of the presentation model based on a hierarchical planner together with graphical resources. The paper also describes an application in the field of hydrology for which the model was developed.
\end{abstract}

\section{KEYWORDS}

Presentation model, multimedia user interface, behavior summarization.

\section{INTRODUCTION}

The general task of management of dynamic systems by means of network of sensors is a type of problem that usually involve teams of operators and decision makers who are interested not only in the interpretations of the evolution of the physical magnitudes, but also in description of possible phenomena and the evolution of these in the time in form of summaries of behavior [3]. Examples of such as dynamic systems are river basins (in hydrology) and road traffic networks. An important task in this context is to give an adequate answer to the presence emergency situations (for example, floods in hydrology or road traffic accidents). Here, it is very important to inform at different and adequate levels of abstraction in order to maximize the number of information receivers and to minimize answer times.
For this purpose, summarization and presentation modeling techniques from the field of Artificial Intelligence can be used to help in this problem [14, 7]. This type of problem requires a solution for presentation that (1) summarizes the evolution of the dynamic system taking into account its structure and relevant events, and (2) dynamically constructs a user-adapted presentation taking into account the communication characteristics (type and importance of the event, user knowledge about the problem, available time, device characteristics, etc.). For this purpose, we have developed the MSB software tool (Multimedia Summarizer of Behavior) that dynamically generates presentations according to the previous needs. Besides an evaluation in different simpler problems, MSB has been successfully applied to the domain of hydrology where a complex presentation model was developed. One of the main contributions of the MSB presentation model is that it captures the characteristics of specific knowledge about how to describe naturally the behavior of dynamic systems. For this purpose, particular discourse patterns and types of applicability conditions have been identified related to models of dynamic systems.

The remaining of the paper is organized as follows. Section 2 describes a general view of the MSB method as a knowledge-based architecture. Section 3 presents the details of the presentation model describing the discourse strategies and the hierarchical planner. Section 4 shows the application of MSB tool to the field of hydrology. At the end of the paper we show a comparative discussion with similar approaches.

\section{THE MSB SUMMARIZER}

The MSB summarizer is a software tool that was developed in the EVIRTUAL project [8]. The goal of the MSB tool is to automatically generate multimedia presentations that summarize the behavior of dynamic systems at appropriate levels of abstraction. This information is presented as concise as possible and is 
accompanied with additional information to facilitate a complete understanding. The information is presented in different modes (text, graphics, animations, etc.) using different devices for reception (computer, mobile phone, fax, etc.).

The method has been designed following a knowledgebased approach (according to recent knowledge engineering methodologies [13]) with a set of general inference steps that use domain specific knowledge. The method performs two main tasks: (1) summarize the most important information (i.e., what to inform) and (2) generate a presentation plan according to the type of enduser and the communication media (how to present the information).

Our method for summarization is based on an explicit representation of the dynamic system (see [8] for more details). The structure of the dynamic system is represented with hierarchies of components with quantities for physical magnitudes and qualitative states. The model includes a simplified view of the system behavior represented with causal relations between quantities with labels such as temporal references about approximated delay and type of influence. Historical values also help to represent information about behavior (e.g., average values, maximum historical values, etc.).

This method produces the summarization tree where the root is the most representative event and the branches include aggregated and less relevant states. Once the relevant information to be reported to the operator is determined (what to inform) it is necessary to generate an adequate presentation plan (how to present the information).

\section{THE MSB PRESENTATION MODEL}

The presentation model is used by the MSB tool to automatically construct multimedia plans. It includes a particular general strategy of discourse that has been designed to inform about the behavior of the dynamic system. In order to inform about relevant events of a dynamic system, MSB develops a discourse strategy that connect informative components with rhetorical relations (as used in Rhetorical Structure Theory [6]).

For this purpose, we have identified a set of rhetorical relations applicable in our context of dynamic system surveillance (see examples in [9]) following the system representation and our notion of relevance.

To articulate rhetorical relations for a particular discourse the method uses discourse patterns. Each pattern is a template that expresses how to develop a part of the discourse. The total set of discourse patterns is implemented as the knowledge base of a planner.

\subsection{The HTN Planner}

The presentation model is represented as the knowledge base of an HTN planner. According to the terminology of HTN planning (Hierarchical Task Network) [2] the model includes planning-tasks, planning-methods, preconditions and operators. In MSB, each planning-task corresponds to a communication goal (e.g., inform about the details of a relevant event) and each planning-method corresponds to a discourse pattern. A pattern has a set of communication sub-goals that corresponds to the rhetorical relations. Thus, the whole strategy of discourse is viewed as a hierarchy of several levels of communication-goals and candidate discourse patterns. Figure 1 shows a partial example of this hierarchy in the field of hydrology.

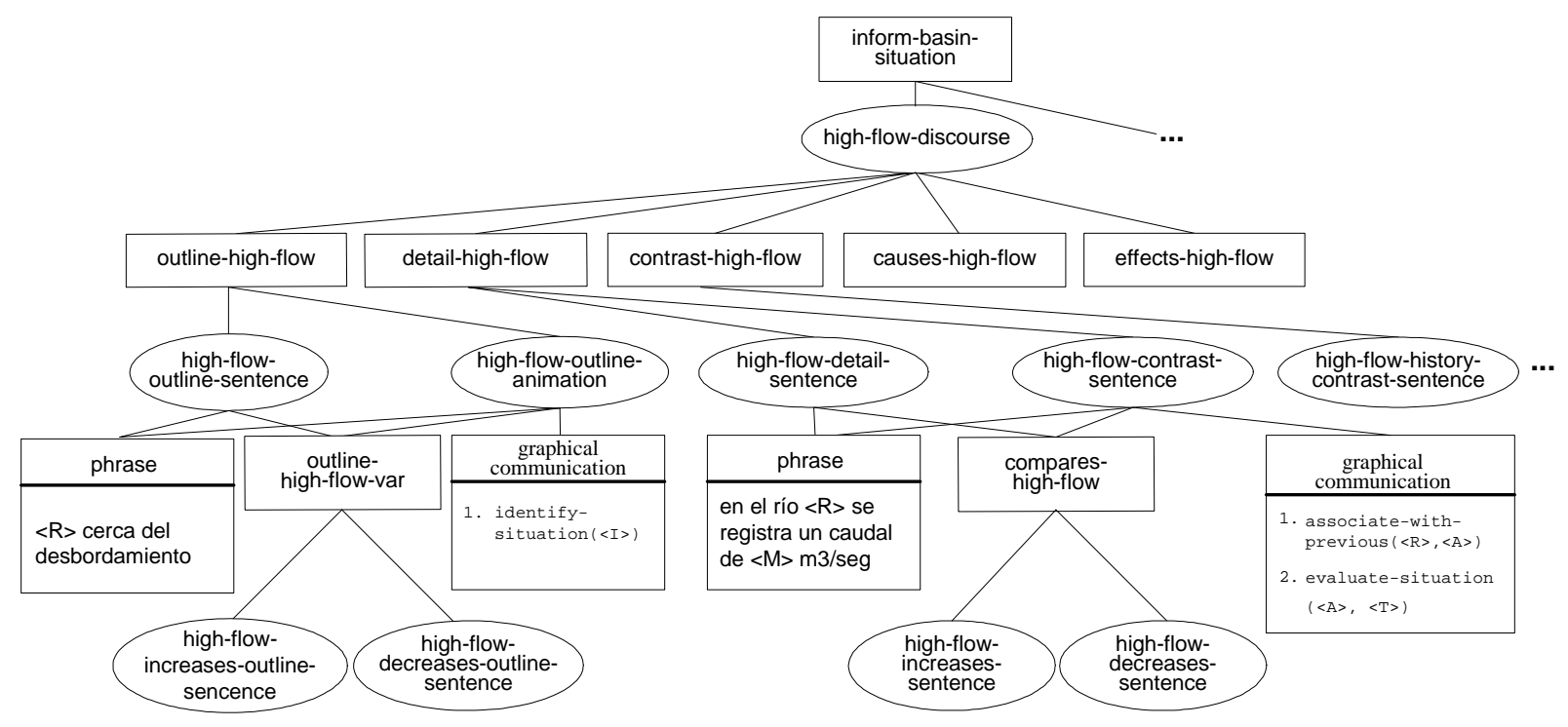

Figure 1: Example (partial) of the HTN-model of discourse patters. In the figure, each rectangle is a communication goal; each ellipse is a discourse pattern and each square a multimedia communicative action (natural language sentence or graphical action). 
In order to select the most appropriate candidate discourse patterns for the same communication goal, the planner includes applicability conditions. The preconditions formulate the applicability conditions of the pattern with the following issues: (1) what, i.e., the characteristics of the event to be informed, (2) who, i.e., the type of user who receives the information (level of background knowledge, available time, etc.), (3) where, i.e., presentation constraints imposed by the communication device (mobile phone, fax, web page, etc.). The preconditions of patterns invoke specialized functions (e.g, causes $(x, y)$ or effects $(x, y)$ ) to gather additional information according the rhetorical relations.

The knowledge base of the planner also includes basic operators for atomic communication goals, i.e., for goals that are not divided into other sub-goals. These operators are implemented as specialized presentation primitives in the form of textual descriptions and parameterized functions that compose text, illustrate with 2D graphics and construct 3D animations. Figure2 presents a possible selection of form-presentation to reach the communicative goal: inform an important flow in rivers of the river basin.

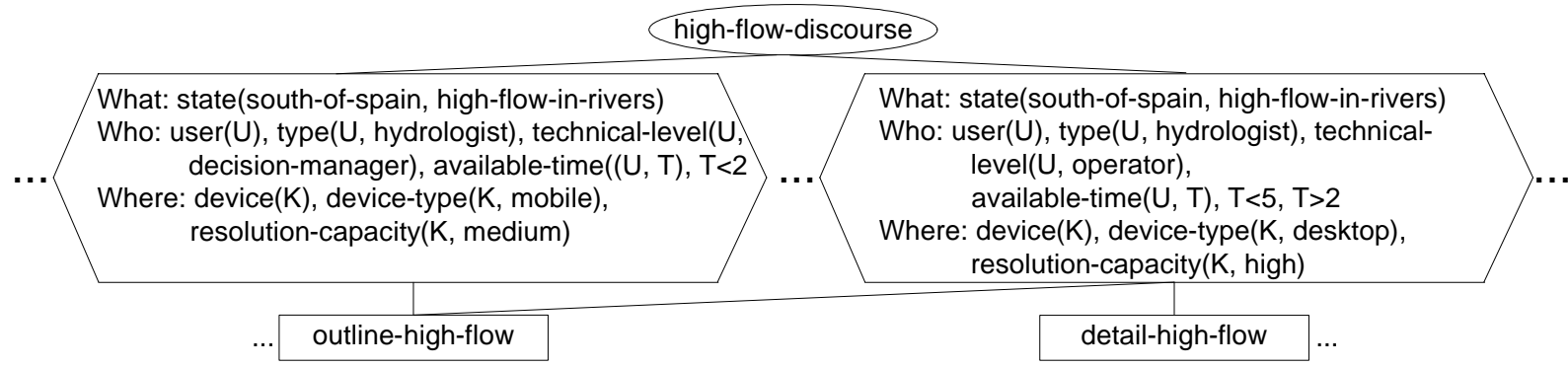

Figure 2: Example of applicability conditions for candidate discourse patterns.

To construct the complete plan the method follows a recursive planning procedure that, in every step performs the following subtasks: (1) select a discourse pattern that satisfies the preconditions, (2) gather additional information according to the preconditions, and (3) refine the communication goal with the sub-goals. The second subtask uses the system model (structure and behavior) together with the aggregation model to construct additional summarization trees for the presentation.

\subsection{The graphical engine for multimedia presentations}

In order to generate the 3D dynamic presentations, MSB communicates with an viewer that includes the presentation strategies and a cartographic database for virtual terrains. This database includes digital elevation models about terrains, vectorial information and ortophotographies. In particular, the planner generates a presentation plan using the HTN knowledge base with discourse patterns and it is communicated to the graphical engine. The graphical engine produces the presentation by using geographic information. In detail, this presentation 3D shows hydrologic information (explanations in natural language) expressed in text [15]. This text presented in information globes and located at geographic places of interest.

\section{APPLICATION TO HYDROLOGY}

MSB was applied to the domain of hydrology to work with real time data of SAIH systems (SAIH: Spanish acronym for Automatic Information System in Hydrology) in Spain. The input data to MSB are hydrologic episodes with a duration of $\mathrm{T}$ hours (for example $\mathrm{T}=24$ hours) with measures of rains, flows and volumes at different points of basins. The system representation includes basic components (e.g., pluviometers, river-sensors, volume-sensors) and other spatial or linear components (e.g., rain area, river and river basin).

These components are related with structural relations (part-of, is-a, etc.). In addition to that, causal relations are used to describe the water behavior from rain components to down-stream components. The basic priority order of interest is: damages $>$ river flow $>$ volume $>$ rain $>$ forecast-of-rain. This order is complemented with detailed heuristic rules for specific cases. The system model is used by the presentation model with the HTN hierarchy that includes 289 discourse patterns according to the general schema (with communicative-goals and communicative-actions).

\section{COMPARISONS WITH RELATED WORK}

Our method for presentation can be compared to techniques of artificial intelligence in the field of presentation models or presentation generation. MSB integrates and extend some general ideas about presentation planning of the WIP project $[7,14]$ for the case of dynamic systems (for example, MSB includes specific discourse patterns and search functions for 
dynamic systems). For the particular case of text composition we follow a template-based approach [11]. Concerning text composition, our solution also presents certain similarities for the case of ILEX [10] although with different model representation, notion of relevance and abstraction method.

Other solutions for presentation generation in the field of meteorology have been proposed. For example, the system of Kerpedjiev [4] follows an approach based [15] but it is restricted to documents and 2D graphics. In this field there are other examples for presentation specialized in natural language generation $[5,1]$. Other applications also generate graphical explanations from data measured by sensors, for example Sumtime [12]. Sumtime is a general application that has been applied in dominions of meteorology, Gas Turbines and medicine. In comparation to this, MSB generates descriptions with animation and multimedia presentations.

\section{CONCLUSIONS}

In summary, the paper presents a model to generate multimedia presentations about the behavior of dynamic systems. The model is used by the MSB software tool (Multimedia Summarizer of Behavior). Our approach contributes with the architecture of a method that identifies different types of knowledge about the dynamic system and model-based strategies for presentation planning that are organized according to rhetorical relations for discourse generation. For this purpose, particular discourse patterns and types of applicability conditions have been identified related to models of dynamic systems.

The advantages of the MSB approach make it appropriate to be used in network contexts to summarize large volumes of quantitative information about complex systems to be reported to a wide range of different types of receivers (operators, decision-makers, other institutions, etc.). For example, MSB has been successfully applied to the domain of hydrology to operate with data from a national hydrologic information infrastructure (the SAIH systems in Spain).

\section{ACKNOWLEDGEMENTS}

This research work has been supported partially by the Ministry of Education and Science of Spain within the EVIRTUAL project (REN2003-09021-C03-02). The Ministry of Environment of Spain (Dirección General del Agua) provided information about the domain in hydrology.

\section{REFERENCES}

1. Bourbeau L., Carcagno D., Goldberg E., Kittredge R., Polguere A.: "Synthesizing Weather Forecasts in an Operational Environment”. In Proc. $13^{\text {th }}$ International Conference COLING, vol. 3, 318-320, Helsinki, August 1990.

2. Ghallab M., Nau D., Traverso P.: “Automated Planning: Theory and Practice”. Morgan Kaufmann, 2004.

3. Kacprzyk J., Wilbik A., and Zadrozny S.: "Linguistic summarization of time series by using the Choquet integral” In the IFSA 2007 World Congress. pp. 284-294. Cancum, Mexico 18-21, June 2007.

4. Kerpedjiev S. M.: “Automatic generation of multimodal weather reports from datasets". Proceedings of the third Conference on Applied Natural Language Processing. Trento, Italy. 48 - 55, 1992.

5. Kittredge R., Polguere A., Goldberg E.: "Synthesizing weather forecasts from formatted data". COLING-86, Proceedings, Bonn, August 1986.

6. Mann W.C., Thompson S.A.: "Rhetorical Structure Theory: Toward a Functional Theory of Text Organization". Text journal, Vol. 8, N. 3, 243-281, 1988.

7. Maybury M. T.: "Generating Summaries from Event Data". Information Processing and Management: an International Journal. Volume 31, Issue 5 (special issue: Summarizing Text) Pages: 735 - 751, September 1995.

8. Molina M., Flores V.: "A knowledge-based approach for automatic generation of summaries of behavior”. Proc. 12th International Conference on Artificial Intelligence: Methodology, Systems, Applications. AIMSA 06. Lecture Notes of Artificial Intelligence. Springer. Varna, Bulgaria, September 2006.

9. Molina M. and Flores V.: "General Adaptative Presentation of Hydrologic Behavior" In Intelligence data Engineering and Automated Learning IDEAL 2006. E. Corchado, H. Yin. V. Botti, C. Fyfe (Eds.). 7th International Conference IDEAL 2006, LNCS 4224. pp. 896-903. Springer-Berlin Heidelberg. Burgos, Spain, September 2006.

10. O'Donnell M.: "Intermixing Multiple Discourse Strategies for Automatic Text Composition". Revista Canaria de Estudios Ingleses (RCEI), No. 40 (April): Special Issue on Intercultural and Textual Approaches to SystemicFunctional Linguistics, 2000.

11. Reiter E.: “NLG vs. Templates". In Fifth European Workshop on Natural Language Generation”. Leiden, 1995.

12. Reiter E,, Sripada S., Hunter J., Yu J., and Davy I.: "Choosing Words in Computer-Generated Weather Forecasts”, Artificial Intelligence, pp.137-169, 2005.

13. Schreiber G., Akkermans H., Anjewierden A., De Hoog R., Shadbolt N., Van de Velde W., Wielinga B.: "Knowledge engineering and management. The CommonKADS methodology” MIT Press, 2000.

14. Wahlster W.: "User and discourse models for multimodal communication". In Sullivan, J. W., Tyler, S. W. \& Sherman, W. editors, Intelligent User Interfaces. New York: ACM Press, S. 45-67, 1991.

15. Wahlster W., André E., Graf W., Rist, T.: "Designing illustrated texts: how language production is influenced by graphics generation", Proceedings of the fifth conference on European chapter of the Association for Computational Linguistics, pp 8-14, April 09-11, Berlin, Germany, 1991. 